\title{
Melody recognition at fast and slow tempos: Effects of age, experience, and familiarity
}

\author{
W. Jay Dowling and James C. Bartlett \\ University of Texas at Dallas, Richardson, Texas \\ ANDREa R. HALPERN \\ Bucknell University, Lewisburg, Pennsylvania \\ AND \\ Melinda W. AndReWs \\ University of Texas at Dallas, Richardson, Texas
}

\begin{abstract}
Eighty-one listeners defined by three age ranges (18-30,31-59, and over 60 years) and three levels of musical experience performed an immediate recognition task requiring the detection of alterations in melodies. On each trial, a brief melody was presented, followed $5 \mathrm{sec}$ later by a test stimulus that either was identical to the target or had two pitches changed, for a same-different judgment. Each melody pair was presented at 0.6 note/sec, 3.0 notes $/ \mathrm{sec}$, or 6.0 notes/sec. Performance was better with familiar melodies than with unfamiliar melodies. Overall performance declined slightly with age and improved substantially with increasing experience, in agreement with earlier results in an identification task. Tempo affected performance on familiar tunes (moderate was best), but not on unfamiliar tunes. We discuss these results in terms of theories of dynamic attending, cognitive slowing, and working memory in aging.
\end{abstract}

A basic characteristic of music is its tempo, or how frequently the primary metric stress occurs. Listeners demonstrate sensitivity to tempo by clapping along with novel music, and they remember the tempos of familiar songs: They confidently set a metronome to their preferred tempo for an imagined familiar song (Halpern, 1988), and when they sing popular songs from their favorite recordings, they match the original tempos fairly closely (Levitin \& Cook, 1996). Memory representations of familiar tunes are flexible, meaning that people can identify tunes across a wide range of tempos (Andrews, Dowling, Bartlett, \& Halpern, 1998; Halpern, 1988; Warren, Gardner, Brubaker, \& Bashford, 1991). However, as tempos become more extreme, encoding becomes increasingly difficult. In the present study, we investigated how age, musical experience, and familiarity with the material modify our ability to process melodies at extreme tempos.

With increasing age, older listeners appear to have difficulty processing very rapid auditory patterns. In our previous study (Andrews et al., 1998), listeners (17-79 years of age) heard familiar melodies played at an extremely fast tempo that became progressively slower until the melodies could be identified. We also started melodies too slow to be identified and gradually speeded them up. In that way, we measured tempo identification thresholds for familiar melodies. Those thresholds agreed in general with those found by Warren et al. (1991), with a range of tempos within which familiar melodies can be recognized going from about $1,670 \mathrm{msec} /$ note $(0.6$ note $/ \mathrm{sec})$ at the slow end to $167 \mathrm{msec} /$ note $(6.0$ notes $/ \mathrm{sec})$ for fast stimuli. We found an effect of age on identification threshold, but only for fast melodies. Age correlated $(r=-.27)$ with thresholds for fast tempos, and not at all with slow tempo thresholds (see Table 1).

These results converge with considerable evidence suggesting that processing speed slows in older adults, leading both to slower reactions in speeded tasks and decrements in other aspects of performance (Park, 2000; Salthouse, 2000). For example, Jastrzembski, Charness, and Vasyukova (2006) found that response times (RTs) of both chess novices and experts lengthened with ageindependent of expertise. Taylor, O'Hara, Mumenthaler, Rosen, and Yesavage (2005) found slowed performance in pilots' comprehension of air-traffic control messages again, independent of expertise. However Morrow et al. (2003) found amelioration of age effects with more experienced pilots in a similar task, which occurred only when the pilots were allowed to take notes while they listened to the messages. These results - along with those of our earlier study - show that cognitive slowing can affect processing even of highly familiar material. Slowing of the neural correlates of cognitive tasks with aging has been 
Table 1

Correlations Between Age and Area Scores, and Experience

(Coded in Four Levels) and Area Scores, in the Present Experiment and in the Identification Task of Andrews et al. (1998)

\begin{tabular}{|c|c|c|c|c|c|c|}
\hline \multirow[b]{3}{*}{ Tempo } & \multirow{2}{*}{\multicolumn{2}{|c|}{$\begin{array}{c}\text { Andrews et al. (1998) } \\
\text { Familiar Tunes }\end{array}$}} & \multicolumn{4}{|c|}{ Present Experiment } \\
\hline & & & \multicolumn{2}{|c|}{ Unfamiliar Tunes } & \multicolumn{2}{|c|}{ Familiar Tunes } \\
\hline & Age & Experience & Age & Experience & Age & Experience \\
\hline Slow & .05 & $.45^{* * *}$ & -.15 & $.49^{* * *}$ & -.09 & $.45^{* * *}$ \\
\hline Moderate & - & - & -.09 & $.42^{* * *}$ & -.06 & $.39^{* * *}$ \\
\hline Fast & $-.27^{* *}$ & $.46^{* * *}$ & $-.29^{* * *}$ & $25^{* * *}$ & $-.20^{*}$ & $.42^{* * *}$ \\
\hline
\end{tabular}

Note-No moderate tempos were tested in Andrews et al. (1998). In both studies, correlations were calculated using age in years and four experience levels: inexperienced, $2-5$ years of musical training, $>5$ years of musical training, and professional. The correlations are based on measures for which higher scores indicate better performance. ${ }^{*} p<$ .10. ${ }^{* *} p<.05$. ${ }^{* * *} p<.01$.

observed with event-related fMRI measures (Rypma, Berger, Genova, Rebbechi, \& D'Esposito, 2005) and with ERP measures (West, Herndon, \& Covell, 2003; West \& Schwarb, 2006).

Such cognitive slowing suggests that older adults might have difficulties in processing fast melodies, as they do for time-compressed speech (Wingfield, Poon, Lombardi, \& Lowe, 1985). A slowdown in processing prevents efficient parsing of elements presented in rapid succession, and some elements may not be well encoded or even processed at all. Pitch-judgment errors made by listeners detecting target tones in rapid temporally interleaved patterns show the effects of hurried and incomplete pitch encoding (Dowling, 1992; Dowling, Lung, \& Herrbold, 1987), leading to poor performance. Numerous studies document the difficulty of speeded tasks with aging, but there are few studies documenting the lack of age-related effects at slow presentation rates. For example, Vaughan, Furukawa, Balasingam, Mortz, and Fausti (2002) found no overall age differences in comprehension of time-expanded speech; all ages showed the same increasing difficulty as the duration of speech was expanded by $20 \%$ to $40 \%$.

One example of the slowing of information processing with age is the slowing of attentional control processes, which is particularly relevant for processing music and speech that are structured in time. In order to track events in the stimulus pattern accurately, we need to "tune" our attentional timing to its temporal structure. In their theory of dynamic attending, Large and Jones (1999) proposed that this temporal tuning of attention is accomplished by the adjustment of internal oscillators to synchronize with the external timing of events. McAuley, Jones, Holub, Johnston, and Miller (2006; see also Baudouin, Vanneste, $\&$ Isingrini, 2004) found that the rate at which event tracking is most accurate slows across the life span. McAuley et al. also found that the preferred tempo - the default setting of the internal oscillators - slowed markedly across the life span, going from 200 beats/min to 86 beats/min ( 3.33 beats $/ \mathrm{sec}$ to 1.43 beats $/ \mathrm{sec}$ ).

In addition to the shift of preferred tempo across the life span, McAuley et al. (2006) also investigated the range of tempos that participants could reproduce reliably. They found a different pattern from the steady slowing of the preferred tempo: The range was relatively small in childhood and relatively large through the middle years, contracting again at older ages. Of particular interest here is that the reduction in range for older participants was mostly in the fast end of the spectrum. This finding agrees with that of Andrews et al. (1998), in that the identification threshold for fast melodies shifted with age, but the identification threshold for slow melodies did not.

Although it is sometimes the case that age-related slowing can be attributed to age-related deficits in working memory (WM), the two forms of cognitive slowing described here - slowed information processing and slowed attentional tracking - are not easily explained based on a more general WM deficit. Although well documented (see, e.g., Bopp \& Verhaeghen, 2005; Verhaeghen, Cerella, \& Basak, 2006), a WM deficit would - in our view-predict decrements in performance at slow tempos as well as at fast. Melodies are encoded primarily by their contour (the pattern of ups and downs) and pitches (represented in terms of scale-step values of the notes; Dowling, 1978, 1986, 1991; Dowling, Tillmann, \& Ayers, 2001). Both contour and pitch encoding involve WM in that successive elements must be actively maintained in memory while the next element is added. Due to the capacity limitations of WM, elements risk being lost during the retention period. A general WM deficit would increase the chances of lost items, especially at slow tempos. Thus, we might have expected deleterious effects of requiring long retention intervals in WM as a consequence of extremely slow stimulus presentation. At very slow rates, earlier elements are likely to be lost from WM by the time later elements are processed, making access to the entire sequence difficult. However, Andrews et al. (1998) found no such effect. Therefore, the cognitive slowing hypothesis appears to provide a better explanation of our earlier results than do the general effects of a WM deficit.

Despite the appeal of the cognitive slowing account, we were concerned that Andrews et al. (1998) used only familiar tunes in their identification paradigm. Doing so may have reduced the load on WM and obscured the effects of a WM deficit that the use of novel melodies might have brought to light. By adding unfamiliar tunes in the present study, we could assess age effects when listeners must encode new information without the cognitive support provided by well-learned musical representations. The 
unfamiliar melodies provide the possibility that requiring the encoding of novel stimulus information will increase the load on WM sufficiently to produce age effects at extremely slow tempos. This finding corresponds with that of Salthouse (1992), that complex materials exacerbate agerelated difficulties in processing - especially in connection with putting greater demands on WM.

In order to vary the familiarity of target tunes, we developed a short-term recognition memory task that does not require the naming of tunes and which can thus be performed with both familiar and unfamiliar tunes. On each trial, the participants heard two tunes in succession and judged them as being either the same or different. The two tunes were presented at very fast, moderate, or very slow tempos (6 notes $/ \mathrm{sec}, 3$ notes $/ \mathrm{sec}$, or 0.6 note/sec, respectively). The fastest and slowest tempos were thus in the neighborhood of the fast and slow thresholds obtained by Andrews et al. (1998) and Warren et al. (1991) as well as of the fastest and slowest viable tempos found by McAuley et al. (2006) for older participants. Listeners had to discriminate exact repetitions of targets from highly similar lures in which the melodic-rhythmic contours were preserved but in which the pitches of two notes were changed. Posing listeners a different task should provide converging evidence bearing on the earlier results, as well as extend our understanding of the effects of aging on the speed of auditory processing - in particular in music perception.

An enduring issue in cognitive aging is how effects related to aging might differ from those related to expertise (see, e.g., Meinz, 2000). Hence, we designed our study to examine the effects of musical experience and of age. Expertise effects are, of course, found in many domains, often most notably in tasks that closely match the actual work done by experts (Morrow, Leirer, Altieri, \& Fitzsimmons, 1994). With musical materials, whether one finds effects of expertise often depends on the particular task that is used (Dowling \& Tillmann, 2004). We have frequently (but not always) found that musicians exceed nonmusicians in musical tasks when the task requires the perceptual encoding of particularly musical elements rather than general auditory processing (Halpern \& Bartlett, 2002). Songs in Andrews et al. (1998) were highly familiar children's, folk, holiday, and patriotic tunes, and their identification might not involve great expertise. Nevertheless, we found a large effect of experience, which correlated at about $r=$ .45 , with performance at both slow and fast thresholds (see Table 1). This result implies that experts are better both at parsing very fast stimulus streams and in holding elements in WM as they arrive very slowly, to build up a pattern. Relevant to this last point, van Zuijen, Sussman, Winkler, Näätänen, and Tervaniemi (2004) showed that musicians are better than nonmusicians in integrating a final element with preceding elements in a sequence, as shown by EEG mismatch negativity (MMN) to a deviant pattern element. Since MMN is considered to indicate a preattentive process (in fact, van Zuijen et al.'s listeners were instructed to ignore the sounds), the ability to group sounds may be an early automatic process useful in explicit tune identification. If so, one would expect to see the effect with unfamiliar melodies as well as with familiar melodies. Indeed, since musicians presumably have more experience than do nonmusicians with encoding novel musical materials, we might find an even larger effect of experience with unfamiliar than with familiar tunes.

In addition to addressing the possibility of effects of expertise, including both familiar and unfamiliar tunes allows us to examine whether familiarity of materials can mitigate age-related declines. Our previous work has rarely found such an interaction (Halpern \& Bartlett, 2002), so we did not predict this in the present study, although we have not often used such speed-sensitive tasks. We also were interested in the interaction of tempo and familiarity. Our thinking was that very fast or very slow tempos might make the encoding of unfamiliar tunes especially difficult. This is because comparing unfamiliar tunes with each other requires precise attention to each note in both the standard and comparison stimulus on a trial, which presumably puts greater demands on encoding processes than does the task of comparing an incoming pattern with a well-learned internal representation.

To summarize, we asked older, middle-aged, and younger people with high, medium, or low levels of musical training to compare one melody with another for a same-different judgment. The melodies were at fast, medium, or slow tempos, and they were familiar or unfamiliar. We predicted moderate age effects at the fast but not at the slow tempos, with the possibility of stronger age effects with unfamiliar melodies. We predicted large experience effects for slow and fast melodies, possibly larger with unfamiliar tunes. Tempo was predicted to affect unfamiliar more than familiar tunes; however, on the basis of prior findings, we did not expect interactions between age and experience or between age and familiarity.

\section{METHOD}

\section{Participants}

Eighty-one listeners served in individual and small-group sessions. Listeners were either paid $\$ 10.00$ for the approximately 40-min session, or - if they were students - received class credit. There were nine listeners in each of the nine cells, defined by three age ranges and three levels of experience. The age groups were: young (17-30 years, $M=23.6$ years), middle (31-59 years, $M=$ 37.4 years), and older (60-79 years, $M=68.3$ years). Listeners were grouped by musical experience on the basis of a brief questionnaire on which they were asked to specify the ages at which they had particular musical training and performance experience. The classification by musical experience was as follows: inexperienced (up to 2 years of formal musical training), moderately experienced (between 2 and 10 years of formal musical training, but not professional), and professional musicians (who had been paid to work as a musician during some part of their life, and who generally had much more than 10 years of experience). Inexperienced listeners averaged 0.2 years of music lessons, and moderately experienced listeners averaged 5.3 years (typically lessons taken between the ages of 8 and 20). Professionals had generally been practicing music actively and continuously from the age of 6 or so and were generally still active. Listeners were recruited by advertisements in the community and by personal contacts, as well as from the psychology student research pool at the University of Texas at Dallas and Bucknell University.

Older listeners received a brief audiometric screening involving the measurement of pure-tone thresholds for four points between 250 and $8000 \mathrm{~Hz}$. All screened participants had age-normal hearing in at least one ear. Two participants could not be screened because 
they wore a hearing aid. In those cases, the experimenter adjusted the intensity of the stimuli so that the listener heard them clearly at a comfortable level. The performance of those 2 listeners was well within the range of scores within their age group.

All listeners completed a vocabulary test based on the Wechsler Adult Intelligence Scale (Wechsler, 1981). The vocabulary test provided a measure of cognitive performance dissimilar to the tasks under investigation (as in Blanchet, Belleville, \& Peretz, 2006). A 3 (ages) $\times 3$ (experience levels) ANOVA on vocabulary performance disclosed only a main effect of age, with the youngest and oldest participants performing about equally well (means of 23.1 and 25.4 points, respectively, out of 40) and the middle-aged group performing somewhat better (28.9 points). Thus, age-related declines in the music tasks could not be attributed to verbal impairment.

\section{Stimuli and Procedure}

The stimuli were produced by a Yamaha PSR-500 synthesizer using its acoustic piano voice and were played legato so that there was little empty space between the notes, but the longer notes faded noticeably before the onset of the succeeding note. The synthesizer was under the control (via MIDI interface) of an IBM-compatible computer running Cakewalk software. Stimuli were recorded on tape and presented to listeners at comfortable levels over high-quality stereophonic equipment.

There were 60 trials. On each trial, a pair of melodies that were either familiar to the listeners or not was presented. The 30 familiar melodies were chosen on the basis of familiarity judgments by listeners over a wide range of ages in previous experiments. The familiar melodies included such tunes as "Over the Rainbow," "Frosty the Snowman," "Take Me Out to the Ball Game," and "When the Saints Go Marching In," as well as other highly familiar folk, nursery, and patriotic tunes. The 30 unfamiliar melodies were selected from McColl and Seeger's (1977) Travellers'Songs From England and Scotland. Melodies were selected that were stylistically similar to the familiar songs; that is, they were unambiguously in a major or minor mode and of fairly regular meter. The first two phrases or so of each melody were used with their natural rhythms for a length of between 11 and 21 notes. The tempos of the melodies were normalized to produce presentation rates of $0.6,3.0$, and 6.0 notes $/ \mathrm{sec}$ in the slow, moderate, and fast conditions, respectively (giving average note durations of $1,667,333$, and $167 \mathrm{msec} /$ note). We controlled presentation rates in terms of notes per second for each song. The various songs had different densities of notes averaging 0.95 notes/ beat and, hence, they had slightly different tempos. The average tempos were $37.9,189.5$, and 378.9 beats $/ \mathrm{min}$, and stimuli ranged in length from 2 to $35 \mathrm{sec}$. The melodies were assigned randomly to tempo conditions. The two melodies on a trial were separated by an interstimulus interval of approximately $5 \mathrm{sec}$. A response interval of $8 \mathrm{sec}$ followed the melody pair. Familiar and unfamiliar melodies were intermingled in the session of 60 trials.

The second melody on each trial was either an exact repetition of the first or a repetition in which two notes in the middle of the melody were changed. We produced altered comparison melodies by changing two pitches in the middle of the song to other diatonic pitches in order to preserve the pitch contour (the pattern of ups and downs) and the rhythm. We avoided changing strongly accented notes. The listener's task was to say whether the two melodies were exactly the same or not. This task was explained with examples drawn from highly familiar tunes in which the changes were obvious. Listeners responded by writing their responses using a six-point confidence level scale with the categories Very sure same, Sure same, Same, Different, Sure different, and Very sure different.

We constructed three versions of the list, and, for purposes of counterbalancing, a third of the listeners in each group received different versions. The main goal of counterbalancing was to ensure that equal numbers of listeners received lists in which a given melody was tested at different tempos so that observed effects of tempo could not be due to the memorability of particular items. In order to generate each list, the original 60 melody pairs were assigned randomly to tempo conditions, with the constraint that no pair could be assigned to a tempo condition to which it was assigned in another list.

Listeners completed the brief questionnaire concerning musical experience and performed the 60 trials of the session in about $35-40 \mathrm{~min}$.

\section{RESULTS}

The proportions of hits and false alarms at the five response criteria defined by the six confidence-level categories were converted to area under the memory operating characteristic, an estimate of the unbiased proportion correct in which chance is .50 (Swets, 1973). The area score provides a better measure of performance than does, for example, $d^{\prime}$ based on the criterion between responses 3 and 4 , because it preserves more response information and over the years has proved to be uncorrelated with measures of bias (unlike $d^{\prime}$-see Dowling, Kwak, \& Andrews, 1995, and Verde, Macmillan, \& Rotello, 2006). Those area scores were subjected to a 3 (ages) $\times 3$ (experience levels) $\times 2$ (familiarity conditions) $\times 3$ (tempo conditions) ANOVA in which age and experience were compared between groups and familiarity and tempo were compared within groups. We calculated $c$ as a measure of response bias on the basis of hit and false-alarm rates derived from the criterion cut between 3 and 4 on the confidence-level scale (i.e., between different responses and same responses; $c$ is the $z$-score distance to the criterion from the symmetrically placed indifference criterion at which false alarms equal misses; higher values of $c$ indicate a more conservative criterion in the sense of one being less likely to respond same).

In the ANOVA on area scores, the effect of age was significant $\left[F(2,72)=3.18, p<.05, r^{2}=.02\right]$, with areas of $0.74,0.76$, and 0.69 for young, medium, and old groups, respectively. The effect of experience was considerably larger $\left[F(2,72)=18.69, p<.001, r^{2}=.10\right]$, with areas of 0.66 , 0.72 , and 0.83 , going from inexperienced to professional (with all differences between adjacent levels of expertise being found significant by a Tukey HSD test). Age and experience did not even remotely interact $[F(4,72)=0.18]$.

Figure 1 shows the results for the familiarity and tempo conditions. The effect of familiarity was significant $\left[F(1,72)=227.97, p<.001, r^{2}=.23\right]$, with better performance on the familiar items. The effect of tempo was significant $\left[F(2,144)=14.34, p<.001, r^{2}=.03\right]$, as was the familiarity $\times$ tempo interaction $[F(2,144)=12.76$, $\left.p<.001, r^{2}=.02\right]$. Tempo had a clear effect on the familiar items, with moderate tempos being superior to slow, which were superior to fast (all differences confirmed by a Tukey HSD test), but tempo had no effect on the unfamiliar items. No other interactions were significant.

In the ANOVA on $c$ scores measuring response bias, the only significant main effects were those of experience $\left[F(2,72)=3.81, p<.05, r^{2}=.02\right]$, familiarity $[F(1,72)=$ $\left.71.50, p<.001, r^{2}=.11\right]$, and tempo $[F(2,144)=13.09$, $\left.p<.001, r^{2}=.06\right]$. Unfamiliar melodies induced a more conservative criterion $(c=0.52)$ than did familiar $(c=$ -0.18 ); novel materials made listeners less likely to respond same. Slower stimuli induced a more conservative 


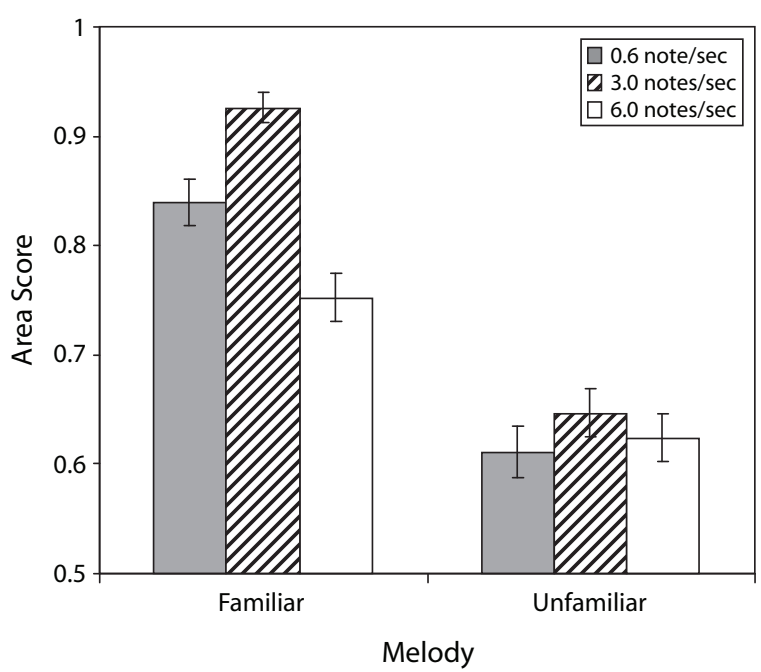

Figure 1. Area scores for familiar and unfamiliar melodies at slow (gray), moderate (hatched), and fast (open) tempos. Error bars are standard errors of the means.

criterion than did fast stimuli $(c=0.51,0.16$, and 0.02 , going from slow to fast). The only other significant effect was the interaction of tempo $\times$ experience $[F(4,144)=$ $\left.2.72, p<.05, r^{2}=.02\right]$. Whereas listeners at all experience levels used conservative criteria at slow tempos $(c=0.43$ to 0.56$)$ and neutral criteria at fast tempos $(c=$ -0.12 to 0.09 ), criteria became increasingly conservative with increasing experience at moderate tempos $(c=$ $-0.20,0.21$, and 0.47 , with increasing experience).

\section{DISCUSSION}

As we expected, performance was best at the young and middle ages, and it was better with more experience and greater familiarity. Moderate tempos conferred an advantage on familiar melodies. As in our previous studies of melody recognition (Andrews et al., 1998; Halpern, Bartlett, \& Dowling, 1995), increasing age led to a decline in performance, but this decline was relatively modest, accounting for $2 \%$ of the variation in the area scores. This result is compatible with both the theory of cognitive slowing in aging and the theory of a slowing of the preferred tempo of internal oscillators with age. Given the absence of an interaction between age and tempo, these results do not permit us to reject the possibility of a WM deficit as one of the sources of the decline in performance with slow melodies with age. These results show that the earlier results were not artifacts of the method. In particular, we now know that the modest size of the age effect in Andrews et al. (1998) was not due to particular task demands, nor to the familiarity of the materials, nor to shifts in response bias with age (none of which were observed). Further, through the use of the recognition method, the results of Andrews et al. are extended to performance at moderate tempos, with the finding that experience-but not age - has an effect there.
The effect of musical experience was much stronger than that of age, accounting for $10 \%$ of the variation in the area scores. The effect of expertise was found for both familiar and unfamiliar melodies across the entire range of tempos, including the moderate tempo. That the effect generalized across familiarity and tempo suggests that it is linked to the development of general musical skills that are applicable to both familiar and unfamiliar tunes at all rates of stimulus presentation. These skills are likely to include effective encoding of pitches of tonal melodies, flexibility in encoding rhythmic patterns at a wide range of tempos, and efficient storage and retrieval of musical materials. As we noted in the introduction, musicians appear to have developed and automatized highly efficient brain mechanisms for the early processing of the auditory information conveyed in musical stimuli, perhaps including the effective use of dynamic attending to tune in to temporal regularities in musical patterns and guide attention accordingly. Musicians may also have more experience in listening for and producing the small variants that composers often apply to musical themes, such as those in classical or jazz forms.

Not surprisingly, performance was better with familiar melodies than with unfamiliar melodies. More interesting was the interaction between familiarity and tempo shown in Figure 1. Contrary to our expectation, performance on familiar melodies was markedly affected by tempo, whereas tempo was not important for processing unfamiliar melodies. This outcome implies that it is not the effect of tempo per se that leads to worse performance for fast and slow stimuli; rather, it is whether the tempo of a wellknown song deviates from its usual, familiar tempo. This effect of tempo with the familiar melodies suggests that hearing a familiar standard stimulus on a trial leads the listener to retrieve a representation of that tune from longterm memory. That representation is likely to be much closer to the moderate tempos than to the fast or the slow tempos used in the present study (Halpern, 1988); hearing a familiar song like "Rudolph the Red-Nosed Reindeer" at an unusually fast or slow tempo could interfere with access to the stored representation as well as reduce its usefulness for comparison with the test stimulus. These considerations would not apply with unfamiliar melodies. The lack of higher order interactions with this tempo/ familiarity pattern suggests that musicians and nonmusicians of all ages use their memory for familiar songs in qualitatively similar ways in this regard.

In order to compare our results directly with those of Andrews et al. (1998), we calculated Pearson correlations between age and performance and between experience and performance in each familiarity and tempo condition (see Table 1; the correlation between age and experience was negligible, $r=-.04)$. The correlations of experience and performance were significant in all tempo $\times$ familiarity conditions. When we averaged across tempos, age was significantly correlated with performance with unfamiliar melodies $(r=-.24)$ and approached significance with familiar melodies $(r=-.15, p<.19)$. In addition, in those cases, the correlations with experience were much stronger: 
$r=.53$ for familiar melodies and $r=.51$ for unfamiliar (both significant at $p<.0001$ ). It is clear that these results do not contradict those of Andrews et al., and, in fact, the correlations in Table 1 show a consistent pattern between the two studies. In particular, the relative strengths of the effects of experience and age are roughly the same in the recognition and identification tasks with fast and slow familiar melodies, with $r^{2}$ in the .18-.21 range for experience and .04-.07 range for age in the fast condition. Adding unfamiliar melodies in the recognition task did not change the pattern of results: Neither age nor experience interacted with familiarity. The correlation of age and performance in the fast condition was significant with unfamiliar tunes and approached significance with familiar tunes $(p<.05$ by a one-tailed test; see Table 1). In view of the pattern of correlations, it is puzzling that age did not interact with tempo in the ANOVA. This lack of an interaction is probably due to the greater sensitivity of the correlations resulting from the use of exact ages rather than age categories. Further research is needed on this point.

A further replication of the results of Andrews et al. (1998) and a number of our previous studies (reviewed in Halpern \& Bartlett, 2002) was the absence of an age $\times$ experience interaction. This was true both for area scores and criterion scores, and the interaction failed to appear even with quite large samples and strongly manipulated variables, hence relatively powerful tests. Although younger age and more experience have beneficial effects on a wide range of music cognition tasks, they do not seem to be interchangeable resources. It may be the case that increasing age affects basic processes such as speed and attentional control, whereas experience confers both domain-specific skills as well as high-level strategies, such as classification and analysis. The preservation of the experience advantage over the life span shows that these specialized skills are maintained in the face of aging effects.

The present study adds to what we learned from the Andrews et al. (1998) study in several ways. First, we wanted to find out whether increasing task complexity by including unfamiliar materials and requiring attention to detail (by changing just two pitches in the middle of the melody) would bring about stronger effects of age, as was suggested by Salthouse's (1992) results with verbal and visual materials. The results show that it did not. In fact, older participants produced the same pattern of behavior with unfamiliar as with familiar tunes, and in a different paradigm. Second, the recognition memory task enabled us to separate response bias from performance measures. Systematic shifts of response bias with age have been observed in other tasks (e.g., in face recognition; Bartlett, 1993). We wanted to be able to rule out response bias shifts with age (or expertise) as a source of our earlier observations of weak age effects, which we in fact did. Finally, the recognition task allowed us to test performance at moderate tempos in addition to very fast and very slow tempos. We did not know from the results of Andrews et al. whether the benefits of experience apply only to the particular demands of tempos restricted to the fast and slow ends of the spectrum. The present results show that experience confers an advantage across the whole range of tempos.
In conclusion, this study confirms our earlier finding that age impairs the processing of melodies, but only to a modest degree. This finding is congruent with cognitive slowing accounts of cognitive aging as well as with the theory of slowing of internal oscillators that guide dynamic attending. This deficit is found with both familiar and unfamiliar melodies, suggesting that cognitive support in the form of familiarity of materials may be only a minor player in this kind of musical processing. Experience effects were large and uniform. That they were not diminished in older people points to preservation of high-level skills across the life span. That they were not enhanced in older people confirms earlier findings that age and experience cannot be traded against one another in accomplishing these tasks. Importantly, the effects of experience and familiarity differed qualitatively. The positive effect of familiarity was greatest at moderate tempos, whereas the positive effect of experience was observed at all tempos. The different effects of familiarity and experience seem to point to two different types of musical knowledge.

Listeners bring to this task both specific knowledge of particular songs and general knowledge of musical structure in their culture - in this case, a knowledge of the tonal framework and the metrical framework and rhythmic patterns of Western European songs (Dowling, 2001). Familiarity with particular songs appears to operate in much the same way for both nonmusician and expert listeners. Familiar tunes are represented in memory in fairly literal ways, in terms both of pitch pattern (Dowling, 1978) and tempo, as shown in the present results in which a moderate presentation tempo was important for familiar (but not for unfamiliar) melodies, as well as in Levitin and Cook's (1996) experiment. In those cases, inexperienced listeners showed qualitatively the same pattern of response as did experienced listeners. In contrast, the knowledge conferred by experience appears to be of much more general applicability, producing equal effects with both familiar and unfamiliar materials. It seems likely that this general knowledge has an implicit impact on perceptual and memory processes involved with attention, encoding, and retrieval, facilitated by more practice with the cognitive frameworks for musical pitch and timing and with the chunking of musical materials. That this knowledge is shared in part by experienced and inexperienced listeners is evident from earlier work demonstrating an implicit knowledge of the tonal framework for the inexperienced (Dowling \& Harwood, 1986). However, it is clear from other studies that pose specific questions about the encoding of melodies that even moderately experienced listeners employ more sophisticated knowledge of musical structure than do the inexperienced (see, e.g., Dowling, 1986), and musicians very likely develop highly sophisticated brain processes both for aiming attention and for the early processing of sensory information.

\section{AUTHOR NOTE}

M.W.A. is now at Waterview Christian Preschool. This research was supported by Grant 1-R01-AG09965 from the National Institute on Aging. We thank Glenn Gesek for assistance in conducting the experiment. Correspondence concerning this article should be addressed to W. J. Dowling, 
School of Behavioral and Brain Sciences, University of Texas at Dallas, Richardson, TX 75083-0688 (e-mail: jdowling@utdallas.edu).

\section{REFERENCES}

Andrews, M. W., Dowling, W. J., Bartlett, J. C., \& Halpern, A. R. (1998). Identification of speeded and slowed familiar melodies by younger, middle-aged, and older musicians and nonmusicians. Psychology \& Aging, 13, 462-471.

BARTLETT, J. C. (1993). Limits on losses in face recognition. In J. Cerella, J. Rybash, W. Hoyer, \& M. L. Commons (Eds.), Adult information processing: Limits on loss (pp. 351-379). San Diego: Academic Press.

Baudouin, A., Vanneste, S., \& Isingrini, M. (2004). Age-related cognitive slowing: The role of spontaneous tempo and processing speed. Experimental Aging Research, 30, 225-239.

Blanchet, S., Belleville, S., \& Peretz, I. (2006). Episodic encoding in normal aging: Attentional resources hypothesis extended to musical material. Aging, Neuropsychology, \& Cognition, 13, 490-502.

Bopp, K. L., \& VerhaEghen, P. (2005). Aging and verbal memory span: A meta-analysis. Journals of Gerontology: Psychological Sciences and Social Sciences, 60B, P223-P233.

Dowling, W. J. (1978). Scale and contour: Two components of a theory of memory for melodies. Psychological Review, 85, 341-354.

Dowling, W. J. (1986). Context effects on melody recognition: Scalestep versus interval representations. Music Perception, 3, 281-296.

Dowling, W. J. (1991). Pitch structure. In P. Howell, R. West, \& I. Cross (Eds.), Representing musical structure (pp. 33-57). London: Academic Press.

Dowling, W. J. (1992). Perceptual grouping, attention and expectancy in listening to music. In J. Sundberg (Ed.), Gluing tones: Grouping in music composition, performance and listening (pp. 77-98). Stockholm: Royal Swedish Academy of Music.

Dowling, W. J. (2001). Perception of music. In E. B. Goldstein (Ed.), Blackwell handbook of perception (pp. 469-498). Oxford: Blackwell.

Dowling, W. J., \& HaRwood, D. L. (1986). Music cognition. Orlando: Academic Press.

Dowling, W. J., KwaK, S.-Y., \& Andrews, M. W. (1995). The time course of recognition of novel melodies. Perception \& Psychophysics, 57, 136-149.

Dowling, W. J., Lung, K. M.-T., \& Herrbold, S. (1987). Aiming attention in pitch and time in the perception of interleaved melodies. Perception \& Psychophysics, 41, 642-656.

Dowling, W. J., \& Tillmann, B. (2004). Les rôles de l'apprentissage perceptif et de l'expertise dans la mémoire des sons, de la musique, et de la poésie. Revue de Neuropsychologie, 14, 169-190.

Dowling, W. J., Tillmann, B., \& Ayers, D. (2001). Memory and the experience of hearing music. Music Perception, 19, 249-276.

Halpern, A. R. (1988). Perceived and imagined tempos of familiar songs. Music Perception, 6, 193-202.

Halpern, A. R., \& Bartlett, J. C. (2002). Aging and memory for music: A review. Psychomusicology, 18, 10-27.

Halpern, A. R., Bartlett, J. C., \& Dowling, W. J. (1995). Aging and experience in the recognition of musical transpositions. Psychology \& Aging, 10, 325-342.

Jastrzembski, T. S., Charness, N., \& Vasyukova, C. (2006). Expertise and age effects on knowledge activation in chess. Psychology \& Aging, 21, 401-405.

Large, E. W., \& Jones, M. R. (1999). The dynamics of attending: How people track time-varying events. Psychological Review, 106, 119-159.

Levitin, D. J., \& Cook, P. R. (1996). Memory for musical tempo: Additional evidence that auditory memory is absolute. Perception \& Psychophysics, 58, 927-935.
McAuley, J. D., Jones, M. R., Holub, S., Johnston, H. M., \& Miller, N. S. (2006). The time of our lives: Life span development of timing and event tracking. Journal of Experimental Psychology: General, 135, 348-367.

McColl, E., \& SEeger, P. (1977). Travellers'songs from England and Scotland. Knoxville: University of Tennessee Press.

MeinZ, E. J. (2000). Experience-based attenuation of age-related differences in music cognition tasks. Psychology \& Aging, 15, 297-312.

Morrow, D. [G.], Leirer, V., Altieri, P., \& Fitzsimmons, C. (1994). When expertise reduces age differences in performance. Psychology \& Aging, 9, 134-148.

Morrow, D. G., Ridolfo, H. E., Menard, W. E., Sanborn, A., StineMorrow, E. A. L., MaGnor, C., ET AL. (2003). Environmental support promotes expertise-based mitigation of age differences on pilot communication tasks. Psychology \& Aging, 18, 268-284.

PARK, D. C. (2000). The basic mechanisms accounting for age-related decline in cognitive function. In D. C. Park \& N. Schwarz (Eds.), Cognitive aging: A primer (pp. 3-21). Philadelphia: Psychology Press.

Rypma, B., Berger, J. S., Genova, H. M., Rebbechi, D., \& D’Esposito, M. (2005). Dissociating age-related changes in cognitive strategy and neural efficiency using event-related fMRI. Cortex, 41, 582-594.

Salthouse, T. A. (1992). Why do adult age differences increase with task complexity? Developmental Psychology, 28, 905-918.

Salthouse, T. A. (2000). Aging and measures of processing speed. Biological Psychology, 54, 35-54.

SwETS, J. A. (1973). The relative operating characteristic in psychology. Science, 182, 990-1000.

Taylor, J. L., O'Hara, R., Mumenthaler, M. S., Rosen, A. C., \& YeSAVAGE, J. A. (2005). Cognitive ability, expertise, and age differences in following air-traffic control instructions. Psychology \& Aging, 20, 117-133.

van Zuijen, T. L., Sussman, E., Winkler, I., NäÄtänen, R., \& TerVANIEMI, M. (2004). Grouping of sequential sounds-An eventrelated potential study comparing musicians and nonmusicians. Journal of Cognitive Neuroscience, 16, 331-338.

Vaughan, N. E., Furukawa, I., Balasingam, N., Mortz, M., \& FAUSTI, S. A. (2002). Time-expanded speech and speech recognition in older adults. Journal of Rehabilitation Research \& Development, 39, 559-566.

Verde, M. F., Macmillan, N. A., \& Rotello, C. M. (2006). Measures of sensitivity based on a single hit rate and false alarm rate: The accuracy, precision, and robustness of $d^{\prime}, A_{z}$, and $A^{\prime}$. Perception \& Psychophysics, 68, 643-654.

Verhaeghen, P., Cerella, J., \& Basak, C. (2006). Aging, task complexity, and efficiency modes: The influence of working memory involvement on age differences in response times for verbal and visuospatial tasks. Aging, Neuropsychology, \& Cognition, 13, 254-280.

WARren, R. M., GARDNer, D. A., BRUbaKer, B. S., \& Bashford, J. A., JR. (1991). Melodic and nonmelodic sequences of tones: Effects of duration on perception. Music Perception, 8, 277-290.

WeChSLER, D. (1981). Wechsler Adult Intelligence Scale (rev. ed.). New York: Psychological Corporation.

West, R., Herndon, R. W., \& Covell, E. (2003). Neural correlates of age-related declines in the formation and realization of delayed intentions. Psychology \& Aging, 18, 461-473.

West, R., \& Schwarb, H. (2006). The influence of aging and frontal function on the neural correlates of regulative and evaluative aspects of cognitive control. Neuropsychology, 20, 468-481.

Wingfield, A., Poon, L. W., Lombardi, L., \& Lowe, D. (1985). Speed of processing in normal aging: Effects of speech rate, linguistic structure, and processing time. Journal of Gerontology, 40, 579-585.

(Manuscript received April 4, 2006; revision accepted for publication October 24, 2007.) 